\title{
Turbulent structure and star formation in a stratified, supernova-driven, interstellar medium
}

\author{
M. K. Ryan Joung ${ }^{1,2} \dagger$ and Mordecai-Mark Mac Low ${ }^{1,2}$ \\ ${ }^{1}$ Department of Astronomy, Columbia University, New York, NY 10027, USA. \\ ${ }^{2}$ Department of Astrophysics, American Museum of Natural History, \\ New York, NY 10024, USA. email: mordecai@amnh.org
}

\begin{abstract}
We report on a study of interstellar turbulence driven by both correlated and isolated supernova explosions. We use three-dimensional hydrodynamic models of a vertically stratified interstellar medium run with the adaptive mesh refinement code Flash at a maximum resolution of $2 \mathrm{pc}$, with a grid size of $0.5 \times 0.5 \times 10 \mathrm{kpc}$. Cold dense clouds form even in the absence of self-gravity due to the collective action of thermal instability and supersonic turbulence. Studying these clouds, we show that it can be misleading to predict physical properties such as the star formation rate or the stellar initial mass function using numerical simulations that do not include self-gravity of the gas. Even if all the gas in turbulently Jeans unstable regions in our simulation is assumed to collapse and form stars in local freefall times, the resulting total collapse rate is significantly lower than the value consistent with the input supernova rate. The amount of mass available for collapse depends on scale, suggesting a simple translation from the density PDF to the stellar IMF may be questionable. Even though the supernova-driven turbulence does produce compressed clouds, it also opposes global collapse. The net effect of supernova-driven turbulence is to inhibit star formation globally by decreasing the amount of mass unstable to gravitational collapse.
\end{abstract}

Keywords. hydrodynamics, ISM: kinematics and dynamics, methods: numerical, turbulence

After briefly describing our numerical model of the ISM, I will concentrate on two results from our work that are most relevant to this meeting: (1) I will report on the density and velocity power spectra of the ISM driven by multiple interacting supernovae (SNe); (2) I will try to determine if SN-driven turbulence enhances or inhibits star formation (see contributions by Vázquez-Semadeni and by Clarke in this volume).

\section{Model}

We set up a 3D stratified atmosphere elongated in the $z$ direction to study the vertical structure. It is a small patch of the galaxy, $(500 \mathrm{pc})^{2}$ in area, and extends from -5 to $+5 \mathrm{kpc}$ in $z$. We use periodic boundary conditions in the $x$ and $y$ directions, and outflow boundary condition at the top and bottom surfaces.

We add instantaneous, localized thermal energy that accounts for both isolated and correlated SNe. (By correlated SNe, I mean superbubbles from OB associations.) The SN rate declines exponentially as the distance from the midplane increases, with different scale heights for type I and type II SNe. The explosion rate is an input parameter, and for our fiducial model, we use the Galactic SN rate.

$\dagger$ Present address: Department of Astrophysical Sciences, Princeton University, Princeton, NJ 08544, USA. email: joung@astro.princeton.edu 

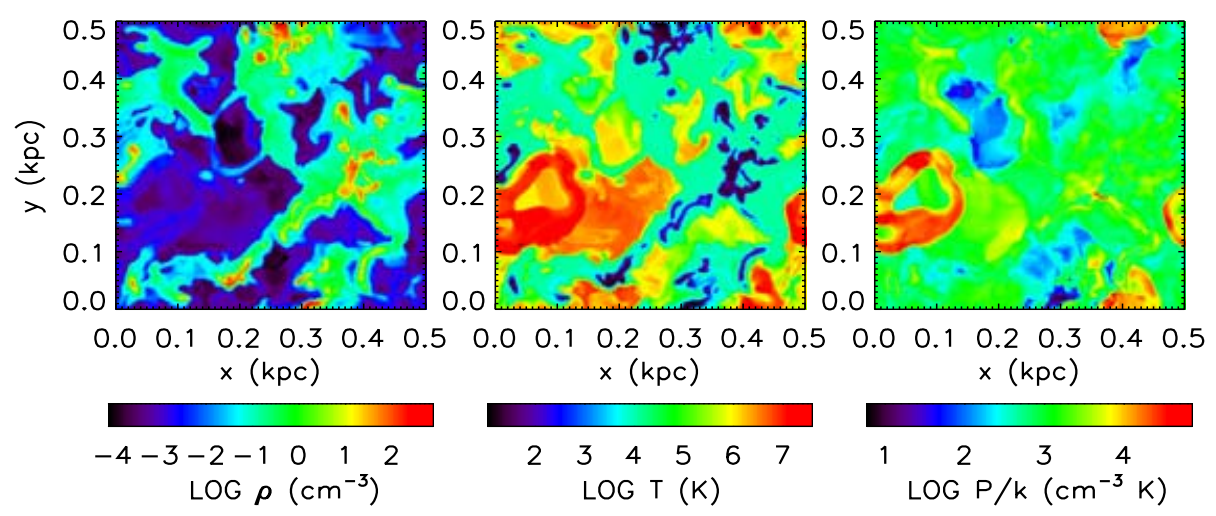

Figure 1. Cuts through the midplane of our model showing distributions of $(a)$ density, (b) temperature, and $(c)$ pressure at $t=79.3 \mathrm{Myr}$.

We include diffuse heating (Wolfire et al. 1995) and radiative cooling (Dalgarno \& McCray 1972; Sutherland \& Dopita 1993) terms and a static vertical gravitational field for stellar and dark halo components (Kuijken \& Gilmore 1989). However, we do not include self-gravity of the gas, magnetic field, or differential rotation of the Galaxy.

Our simulations are performed using Flash (Fryxell et al. 2000). It is a grid-based hydrodynamics code with adaptive mesh refinement (AMR) capability. The AMR allows us to focus resolution only where it is necessary, i.e., where density and pressure gradients are large. We end up maximally resolving the region near the midplane of the galaxy, $|z| \lesssim 300$ pc, because the bulk of the gas and most of the SNe reside there. See Joung \& Mac Low (2006, hereafter JM06) for more details of the model.

\section{Results}

Figure $1(a, b, c)$ shows the density, temperature, and pressure distributions in the midplane. The hot regions, i.e. red and yellow regions in figure $1(b)$, always correspond to relatively low densities. Correlated SNe produce much of the volume occupied by hot gas. Cold clouds, the red, high density regions in figure $1(a)$, are filamentary in shape, and are surrounded by thick layers of warm gas. They have temperatures as low as $10 \mathrm{~K}$, so they resemble molecular clouds observed in the ISM. These clouds form when SN blast waves interact with each other. Note that they are present even though self-gravity of the gas is not included in our model.

Both density and temperature vary by about 7 orders of magnitude, but because of the approximate inverse relationship they have, for most the volume, the thermal pressure varies only by 2 orders of magnitude. The pressure PDF shows that there is as much gas below the average pressure as above it. The variation in pressure is inconsistent with ISM models based on pressure equilibrium between phases (e.g., Field et al. 1969). This implies that we need a more dynamic picture (Mac Low \& Klessen 2004).

Figure $2(a, b)$ shows the density and kinetic energy power spectra from our model. The density spectrum is completely dominated by clumpy cold clouds, because they have orders of magnitude higher densities than the hot or the warm medium.

To characterize the distribution of kinetic energy, people have usually plotted the power spectrum of the velocity field. However, in a strongly compressible medium, a more dynamically meaningful quantity is the power spectrum of $\sqrt{\rho} v$, which I call the 

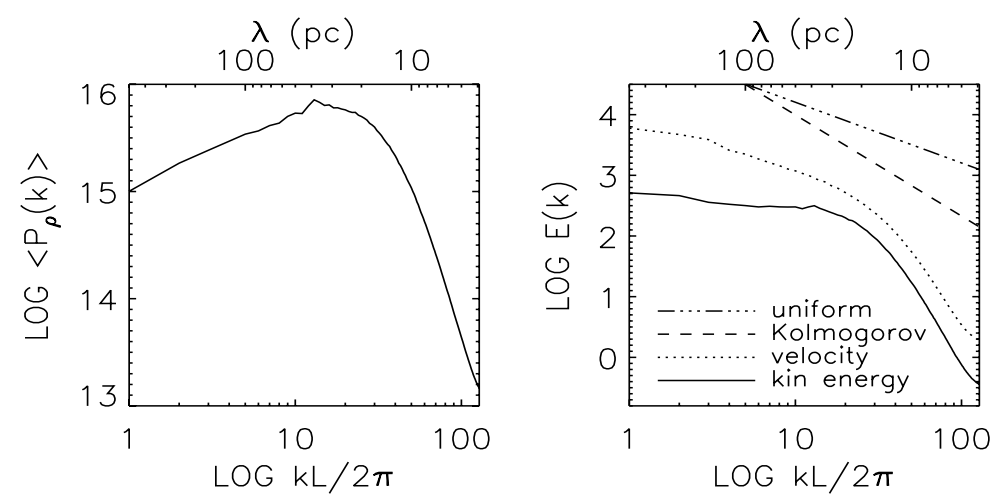

Figure 2. (a) Angle-averaged density power spectrum, displaying a wide peak around $k L / 2 \pi \approx 20$. The box size $L=0.5 \mathrm{kpc}$. (b) Kinetic energy spectrum (solid) and angle-averaged velocity power spectrum (dotted). Because of the highly intermittent density structure, the velocity power spectrum is not parallel to the kinetic energy spectrum, especially at large scales. To guide the eye, two straight lines are plotted: the Kolmogorov energy spectrum (dashed) and the spectrum containing an equal amount of energy per decade (dot-dashed).

kinetic energy spectrum. In a uniform medium, this distinction will be unnecessary. The velocity spectrum does not distinguish between high density and low density regions, and in our case has more power at large scales, so it is slightly steeper than this kinetic energy spectrum. Instead of single driving and dissipation scales and a constant slope of $-5 / 3$ in between, as predicted by Kolmogorov (dashed line in figure $2(b)$ ), the kinetic energy spectrum of a SN-driven ISM (solid curve) shows that there is no single effective driving scale; in fact, we see energy injection over a range of scales, as expected in a medium driven by multiple interacting explosions. Despite that, though, $90 \%$ of the total kinetic energy is contained in scales shortward of 190 pc (JM06). On a side note, let me remind you that our model included only SN driving. Gravitational instability, for example, could have contributed more power on large scales, if we had included it.

Of course, what is actually observable is the column density power spectrum. We have created power spectra of the total gas column density from our ISM simulations and compared them with the two-dimensional power spectrum of the H I column density in the LMC from Elmegreen, Kim, \& Staveley-Smith (2001). Although our spectrum does not have a constant slope, we find that the overall slope is intriguingly close to the value $(-8 / 3)$ measured from the $\mathrm{H}$ I observations.

In our model, cold dense clouds form directly in the turbulent flow. We may ask: what fraction of these clouds are gravitationally unstable, given the density and velocity structures in our model? Although our numerical model does not include self-gravity, it does represent other physical processes reasonably well, such as SN shock compressions or thermal instability, Hence the formation of unstable clouds, if any, must have been induced purely by SN-driven turbulence.

To do this, we took a rectangular region near the midplane, tiled it with smaller cubes, and applied a simple criterion for gravitational collapse, i.e., a modified Jeans criterion (Chandrasekhar 1951) to identify Jeans unstable boxes: $M_{b o x} / M_{J}>1$, where the Jeans length $\lambda_{J}=(\pi / G \bar{\rho})^{1 / 2} \sigma_{t o t}$ and $\sigma_{t o t} \equiv\left({\overline{c_{s}}}^{2}+\frac{1}{3} \sigma^{2}\right)^{1 / 2}$. We also assume that the gas in the unstable boxes turn into stars in the local free-fall time, with some efficiency factor. We repeated this procedure for various box sizes. Then we can compare the total gravitational collapse rate and the input $\mathrm{SN}$ rate (which can be converted to a star formation rate using some reasonable IMF). In steady state, the two rates should be equal. In this way, 


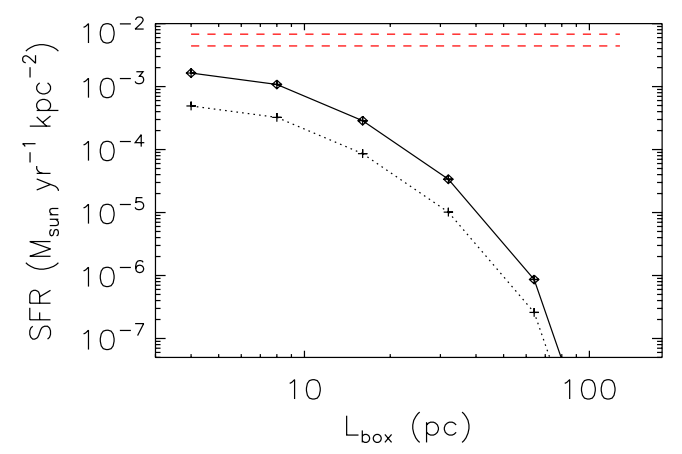

Figure 3. Predicted star formation rate from the model plotted against the subbox sizes used. The dotted line is drawn assuming that $30 \%$ of the mass in Jeans unstable regions turn into stars. The red dashed lines show the star formation rates consistent with the assumed Galactic $\mathrm{SN}$ rate, if 130 or $200 \mathrm{M}_{\odot}$ of stellar mass is required per $\mathrm{SN}$.

we can quantitatively check the validity of our high-resolution simulations as a model for star formation (JM06).

While doing this analysis, we obtained as a by-product a simple relationship between the total velocity dispersion $\left(\sigma_{t o t}\right.$; thermal+turbulent) and the average gas density within each box $(\bar{\rho}): \sigma_{t o t} \propto \bar{\rho}^{-1 / 2}$, which implies interestingly $\bar{\rho} \sigma_{\text {tot }}^{2} \approx$ const. (Joung, Mac Low, $\&$ Bryan, in preparation). But due to the time limit, I will not discuss it here.

Figure 3 displays the result of our simple calculation. For boxes that are bigger than $20 \mathrm{pc}$ on a side, that is, where turbulent motions are resolved, even if we assume all the gas in turbulent Jeans unstable boxes collapses and forms stars within the local freefall time, the resulting star formation rate will remain lower than the value consistent with our input SN rate (red lines). This shows that it may be misleading to use the statistics of turbulent fluctuations to predict physical properties of star-forming regions. The discrepancy arises probably from the neglect of self-gravity of the gas in our model. Hence, SNcompressions alone can indeed trigger star formation, but not all of it, maybe only $\sim 10 \%$ of the total star formation rate, comparable to the estimate by Mizuno (this volume).

In contrast, the initial setup of our simulation was gravitationally stable on all scales. It was, however, out of thermal equilibrium. Without extra stirring from SNe, the gas would have promptly cooled and collapsed into a thin sheet al.ng the midplane, making more gas gravitationally unstable. Therefore, the net effect of SN-driven turbulence in this case was to inhibit collapse globally.

\section{Acknowledgements}

We would like to thank G. Bryan, I. Goldman, Y. Li, C. McKee, and J. Oishi for useful comments and stimulating discussions. M. K. R. J. was supported by an AMNH Graduate Student Fellowship for the duration of this work. M.-M. M. L. acknowledges support by NSF grants AST03-07793 and AST03-07854. The software used in this work was in part developed by the DOE-supported ASCI/Alliance Center for Astrophysical Thermonuclear Flashes at the University of Chicago. Computations were performed at the Pittsburgh Supercomputing Center.

\section{References}

Chandrasekhar S. 1951, Proc. Royal Soc. London A 210, 26 
Dalgarno, A. \& McCray, R.A. 1972, ARA $\& A$ A 10, 375

Elmegreen, B.G., Kim, S. \& Staveley-Smith, L. 2001, ApJ 548, 749

Field, G.B., Goldsmith, D.W. \& Habing, H.J. 1969, ApJ 155, L49

Fryxell, B., Olson, K., Ricker, P., Timmes, F.X., Zimgale, M., et al. 2000, ApJS 131, 273

Joung, M.K.R. \& Mac Low, M.-M. 2006, ApJ in press (JM06)

Kuijken, K. \& Gilmore, G. 1989, MNRAS 239, 605

Mac Low, M.-M. \& Klessen, R.S. 2004, Rev. Mod. Phys. 76, 125

Sutherland, R.S. \& Dopita, M.A. 1993, ApJS 88, 253

Wolfire, M.G., McKee, C.F., Hollenbach, D., Tielens, A.G.G.M. \& Bakes, E.L.O. 1995, ApJ 443, 152

\section{Discussion}

PALOuš: Does $\sigma$ in your modified Jeans mass include bulk motions?

JounG: Yes. For any given subbox, I compute the rms velocity dispersion of all the cells within it. It is actually what I called $\sigma$ in the expression for $\sigma_{t o t}$.

ELMEgREen: What fraction of your supernova remnants look like shells, and do you have clustered supernovae?

JoUnG: I assumed a power-law distribution for the number of supernovae in clusters, following Clarke \& Oey. A small fraction of the supernova remnants do look like shells as you can see in figure $1(b)$, but I have not quantified the fraction.

Goldman: Your kinetic energy power spectrum shows a break around 20-30 pc. Is your power spectrum steeper than the Kolmogorov spectrum on small scales?

JounG: The shape of the kinetic energy power spectrum below about $20 \mathrm{pc}$ is determined by numerics, so the spectrum shortward of $\sim 20$ pc should not be trusted.

NAKAmuRA: You said that turbulence inhibits star formation, but that might change when you include magnetic field in your model.

JounG: Since the turbulence in our model is driven, rather than decaying, it decreases the star formation efficiency, just as Enrique has pointed out. However, I agree that adding magnetic field to the model is important. It is probably the next step one should take. 